\title{
The extragradient-Armijo method for pseudomonotone equilibrium problems and strict pseudocontractions
}

\author{
Pham Ngoc Anh ${ }^{1 *}$ and Nguyen Duc Hien ${ }^{2}$
}

\footnotetext{
* Correspondence: anhpn@ptit.edu. $\mathrm{vn}$

${ }^{1}$ Department of Scientific Fundamentals, Posts and Telecommunications Institute of Technology, Hanoi, Vietnam Full list of author information is available at the end of the article
}

\begin{abstract}
In this article, we present a new iteration method for finding a common element of the set of fixed points of $p$ strict pseudocontractions and the set of solutions of equilibrium problems for pseudomonotone bifunctions without Lipschitz-type continuous conditions. The iterative process is based on the extragradient method and Armijo-type linesearch techniques. We obtain weak convergence theorems for the sequences generated by this process in a real Hilbert space.

AMS 2010 Mathematics Subject Classification: 65 K10; 65 K15; 90 C25; 90 C33.

Keywords: equilibrium problems, pseudomonotone, extragradient method, strict pseudocontractions, fixed point, linesearch
\end{abstract}

\section{Introduction}

Let $C$ be a nonempty closed convex subset of a real Hilbert space $\mathcal{H}$ and $f$ be a bifunction from $C \times C$ to $\mathcal{R}$. We consider the following equilibrium problems (shortly $E P(f$, C)):

$$
\text { Find } x^{*} \in C \text { such that } f\left(x^{*}, y\right) \geq 0 \quad \text { for all } y \in C \text {. }
$$

The set of solutions of Problem $E P(f, C)$ is denoted by $\operatorname{Sol}(f, C)$. These problems apprear frequently in many practical problems arising, for instance, physics, engineering, game theory, transportation, economics and network, and become an attractive field for many researchers both theory and applications (see [1-6]). The bifunction $f$ is called

- monotone if

$$
f(x, y)+f(y, x) \leq 0, \quad \forall x, y \in C ;
$$

- pseudomonotone if

$$
f(x, y) \geq 0 \Rightarrow f(y, x) \leq 0, \quad \forall x, y \in C ;
$$

- Lipschitz-type continuous with constants $c_{1}>0$ and $c_{2}>0$ if

$$
f(x, y)+f(y, z) \geq f(x, z)-c_{1}\|x-y\|^{2}-c_{2}\|y-z\|^{2}, \quad \forall x, y \in C .
$$

It is clear that every monotone bifunction $f$ is pseudomonotone.

(c) 2012 Anh and Hien; licensee Springer. This is an Open Access article distributed under the terms of the Creative Commons Attribution License (http://creativecommons.org/licenses/by/2.0), which permits unrestricted use, distribution, and reproduction in any medium, provided the original work is properly cited. 
Let $C$ be a nonempty closed convex subset of $\mathcal{H}$. A self-mapping $S: C \rightarrow C$ is called a strict pseudocontraction if there exists a constant $0 \leq L<1$ such that

$$
\|S(x)-S(y)\|^{2} \leq\|x-y\|^{2}+L\|(I-S)(x)-(I-S)(y)\|^{2}, \quad \forall x, y \in C,
$$

where $I$ is the identity mapping on $C$. The set of fixed points of $S$ is denoted by Fix $(S)$. The following proposition lists some useful properties for strict pseudocontractions.

Proposition 1.1 [7]Let $C$ be a nonempty closed convex subset of a real Hilbert space $\mathcal{H}, S: C \rightarrow C$ be a L-strict pseudocontraction and for each $i=1, \ldots, p, S_{i}: C \rightarrow C$ is a $L_{i}$-strict pseudocontraction for some $0 \leq L_{i}<1$. Then,

(a) S satisfies the Lipschitz condition

$$
\|S(x)-S(y)\| \leq \frac{1+L}{1-L}\|x-y\|, \quad \forall x, y \in C ;
$$

(b) $I-S$ is demiclosed at 0 . That is, if $\left\{x^{n}\right\}$ is a sequence in $C$ such that $x^{n} \rightarrow \bar{x}$ and (I $-S)\left(x^{n}\right) \rightarrow 0$, then $(I-S)(\bar{x})=0$;

(c) the fixed point set $\operatorname{Fix}(S)$ is closed and convex;

(d) if $\lambda_{i}>0$ and $\sum_{i=1}^{p} \lambda_{i}=1$, then $\sum_{i=1}^{p} \lambda_{i} S_{i}$ is a $\bar{L}_{\text {-strict }}$ pseudocontraction with $\bar{L}=\max \left\{L_{i}: 1 \leq i \leq L\right\}$

(e) if $\lambda_{i}$ is given as in $(d)$ and $\left\{S_{i}: i=1, \ldots, p\right\}$ has a common fixed point, then

$$
\operatorname{Fix}\left(\sum_{i=1}^{p} \lambda_{i} S_{i}\right)=\bigcap_{i=1}^{p} \operatorname{Fix}\left(S_{i}\right) .
$$

For finding a common fixed point of $p$ strict pseudocontractions $\left\{S_{i}\right\}_{i=1}^{p}$, Mastroeni [5] introduced an iterative algorithm in a real Hilbert space. Let sequences $\left\{x^{n}\right\}$ be defined by

$$
x^{n+1}=\alpha_{n} x^{n}+\left(1-\alpha_{n}\right) \sum_{i=1}^{p} \lambda_{n, i} S_{i}\left(x^{n}\right),
$$

Under appropriate assumptions on the sequence $\left\{\lambda_{n, i}\right\}$, the authors showed that the sequence $\left\{x^{n}\right\}$ converges weakly to the same point $\bar{x} \in \cap_{i=1}^{p} \operatorname{Fix}\left(S_{i}\right)$.

For obtaining a common element of set of solutions of Problem $E P(f, C)$ and the set of fixed points of a nonexpansive mapping $S$ in a real Hilbert space $\mathcal{H}$, Takahashi and Takahashi [8] first introduced an iterative scheme by the viscosity approximation method. The sequence $\left\{x^{n}\right\}$ is defined by

$$
\left\{\begin{array}{l}
x^{0} \in \mathcal{H}, \\
f\left(u^{n}, y\right)+\frac{1}{r_{n}}\left\langle y-u^{n}, u^{n}-x^{n}\right\rangle \geq 0, \quad \forall y \in C, \\
x^{n+1}=\alpha_{n} g\left(x^{n}\right)+\left(1-\alpha_{n}\right) T\left(u^{n}\right), \quad \forall n \geq 0 .
\end{array}\right.
$$

The authors showed that under certain conditions over $\left\{\alpha_{n}\right\}$ and $\left\{r_{n}\right\}$, sequences $\left\{x^{n}\right\}$ and $\left\{u^{n}\right\}$ converge strongly to $z=\operatorname{Pr}_{\mathrm{Fix}(T) \cap \operatorname{Sol}(f, C)}(g(z))$, where $\operatorname{Pr}_{C}$ is denoted the projection on $C$ and $g: C \rightarrow C$ is contractive, i.e., ||$g(x)-g(y) \| \leq \delta|| x-y||$ for all $x, y \in C$.

Recently, for finding a common element of the set of common fixed points of a strict pseudocontraction sequence $\left\{\hat{S}_{i}\right\}$ and the set of solutions of Problem EP $(f, C)$, Chen et al. [9] proposed new iterative scheme in a real Hilbert space. Let sequences $\left\{x^{n}\right\},\left\{y^{n}\right\}$ 
and $\left\{z^{n}\right\}$ be defined by

$$
\left\{\begin{array}{l}
x^{0} \in C, \\
y^{n}=\alpha_{n} x^{n}+\left(1-\alpha_{n}\right) \hat{S}_{n}\left(x^{n}\right), \\
f\left(z^{n}, y\right)+\frac{1}{r_{n}}\left\langle y-z^{n}, z^{n}-y^{n}\right\rangle \geq 0, \quad \forall y \in C, \\
C_{n}=\left\{v \in C:\left\|z^{n}-v\right\| \leq\left\|x^{n}-v\right\|\right\}, \\
x^{n+1}=\operatorname{Pr}_{C_{n}}\left(x^{0}\right) .
\end{array}\right.
$$

Then, they showed that under certain appropriate conditions imposed on $\left\{\alpha_{n}\right\}$ and $\left\{r_{n}\right\}$, the sequences $\left\{x^{n}\right\},\left\{y^{n}\right\}$ and $\left\{z^{n}\right\}$ converge strongly to $\operatorname{Pr}_{\operatorname{Fix}(S) \cap S o l(f, C)}\left(x^{0}\right)$, where $S$ is a mapping of $C$ into itself defined by $S(x)=\lim _{n \rightarrow \infty} \hat{S}_{n}(x)$ for all $x \in C$.

There exist some another solution methods for finding a common element of the set of solutions of Problem $E P(f, C)$ and $\cap_{i=1}^{p} \operatorname{Fix}\left(S_{i}\right)$ (see [3,10-19]). Most of these algorithms are based on solving approximation equilibrium problems for strongly monotone or monotone and Lipschitz-type continuous bifunctions on $C$. In this article, we introduce a new iteration method for finding a common element of the set of common fixed points of $p$ strict pseudocontractions and the set of solutions of equilibrium problems for pseudomonotone bifunctions. The fundamental difference here is that at each iteration $n$, we only solve a strongly convex problem and perform a projection on $C$. The iterative process is based on the extragradient method and Armijo-type linesearch techniques. We obtain weak convergence theorems for sequences generated by this process in a real Hilbert space $\mathcal{H}$.

\section{Preliminaries}

Let $C$ be a nonempty closed convex subset of a real Hilbert space $\mathcal{H}$. For each point $x \in \mathcal{H}$, there exists the unique nearest point in $C$, denoted by $\operatorname{Pr}_{C}(x)$, such that

$$
\left\|x-\operatorname{Pr}_{C}(x)\right\| \leq\|x-y\|, \quad \forall y \in C .
$$

$\operatorname{Pr}_{C}$ is called the metric projection on $C$. We know that $\operatorname{Pr}_{C}$ is a nonexpansive mapping on $C$. It is also known that $\operatorname{Pr}_{C}$ is characterized by the following properties

$$
\operatorname{Pr}_{C}(x) \in C, \quad\left\langle x-\operatorname{Pr}_{C}(x), \operatorname{Pr}_{C}(x)-y\right\rangle \geq 0,
$$

for all $x \in \mathcal{H}, y \in C$. In the context of the convex optimization, it is also known that if $g: C \rightarrow \mathcal{R}$ is convex and subdifferentiable on $C$, then $\bar{x}$ is a solution to the following convex problem

$$
\min \{g(x): x \in C\}
$$

if and only if $0 \in \partial g(\bar{x})+N_{C}(\bar{x})$, where $N_{C}(\bar{x})$ is out normal cone at $\bar{x}$ on $C$ and $\partial g(\cdot)$ denotes the subdifferential of $g$ (see [20]).

Now we are in a position to describe the extragradient-Armijo algorithm for finding a common element of $\cap_{i=1}^{p} \operatorname{Fix}\left(S_{i}\right) \cap \operatorname{Sol}(f, C)$.

Algorithm 2.1 Given a tolerance $\varepsilon>0$. Choose $x^{0} \in C, k=0, \gamma \in(0,1), 0<\sigma<\frac{\beta}{2}$ and positive sequences $\left\{\lambda_{n, i}\right\}$ and $\left\{\alpha_{n}\right\}$ satisfy the conditions:

$$
\left\{\begin{array}{l}
\left\{\alpha_{n}\right\} \subset[a, b] \subset(\bar{L}, 1) \text { where } \bar{L}:=\max \left\{L_{i}: \quad 1 \leq i \leq p\right\} \\
\sum_{i=1}^{p} \lambda_{n, i}=1 \text { for all } n \geq 1, \lim _{n \rightarrow \infty} \lambda_{n, i}=\lambda_{i} \in(0,1) \text { for all } i=1, \ldots, p, \sum_{i=1}^{p} \lambda_{i}=1 .
\end{array}\right.
$$


Step 1. Solve the strongly convex problem

$$
y^{n}=\operatorname{argmin}\left\{f\left(x^{n}, y\right)+\frac{\beta}{2}\left\|y-x^{n}\right\|^{2}: y \in C\right\} \text { and setr }\left(x^{n}\right)=x^{n}-y^{n} .
$$

If $\left\|r\left(x^{n}\right)\right\| \neq 0$ then go to Step 2. Otherwise, set $w^{n}=x^{n}$ and go to Step 3.

Step 2. (Armijo-type linesearch techniques) Find the smallest positive integer number $m_{n}$ such that

$$
f\left(x^{n}-\gamma^{m_{n}} r\left(x^{n}\right), y^{n}\right) \leq-\sigma\left\|r\left(x^{n}\right)\right\|^{2} .
$$

Compute

$$
w^{n}=\operatorname{Pr}_{C \cap H_{n}}\left(x^{n}\right),
$$

where

$$
z^{n}=x^{n}-\gamma^{m_{n}} r\left(x^{n}\right), v^{n} \in \partial_{2} f\left(z^{k}, z^{k}\right)
$$

and

$H_{n}=\left\{x \in \mathcal{H}:\left\langle v^{n}, x-z^{n}\right\rangle \leq 0\right\}$, and go to Step 3.

Step 3. Compute

$$
x^{n+1}=\alpha_{n} w^{n}+\left(1-\alpha_{n}\right) \sum_{i=1}^{p} \lambda_{n, i} S_{i}\left(w^{n}\right) .
$$

Increase $n$ by 1 and go back to Step 1 .

Remark 2.2 If $\left\|r\left(x^{n}\right)\right\|=0$ then $x^{n}$ is a solution to Problem $E P(f, C)$ but it may be not a common fixed point of $\left\{S_{i}\right\}_{i=1}^{p}$.

Indeed, $\left\|r\left(x^{n}\right)\right\|=0$, i.e., $x^{n}$ is the unique solution to

$$
\min \left\{f\left(x^{n}, y\right)+\frac{\beta}{2}\left\|y-x^{n}\right\|^{2}: y \in C\right\} .
$$

Then

$$
0 \in \partial_{2} f\left(x^{n}, x^{n}\right)+N_{C}\left(x^{n}\right) .
$$

Hence

$$
\left\langle v^{n}, x-x^{n}\right\rangle \geq 0, \quad \forall x \in C, \quad v^{n} \in \partial_{2} f\left(x^{n}, x^{n}\right) .
$$

Combining this inequality with $f\left(x^{n}, x^{n}\right)=0$ and the convexity of $f\left(x^{n}, \cdot\right)$, i.e.,

$$
f\left(x^{n}, x\right)-f\left(x^{n}, x^{n}\right) \geq\left\langle v^{n}, x-x^{n}\right\rangle, \forall x \in C, v^{n} \in \partial_{2} f\left(x^{n}, x^{n}\right),
$$

we have $f\left(x^{n}, x\right) \geq 0$ for all $x \in C$. It means that $x^{n}$ is a solution to Problem $E P(f, C)$.

\section{Convergence results}

In this section, we show the convergence of the sequences $\left\{\mathrm{x}^{n}\right\},\left\{y^{n}\right\}$ and $\left\{w^{n}\right\}$ defined by Algorithm 2.1 is based on the extragradient method and Armijo-type linesearch techniques which solves the problem of finding a common element of two sets $\cap_{i=1}^{p} \operatorname{Fix}\left(S_{i}\right)$ and Sol $(f, C)$. To prove it's convergence, we need the following preparatory result.

Lemma 3.1 [21] Let $C$ be a nonempty closed convex subset of a real Hilbert space $\mathcal{H}$. Suppose that, for all $u \in C$, the sequence $\left\{x^{n}\right\}$ satisfies 


$$
\left\|x^{n+1}-u\right\| \leq\left\|x^{n}-u\right\|, \forall n \geq 0 .
$$

Then, the sequence $\left\{\operatorname{Pr}_{C}\left(x^{n}\right)\right\}$ converges strongly to $\bar{x} \in C$.

We now state and prove the convergence of the proposed iteration method.

Theorem 3.2 Let $C$ be a nonempty closed convex subset of $\mathcal{H}, S_{i}: C \rightarrow C$ be a $L_{i^{-}}$ Lipschitz pseudocontractions for all $i=1, \ldots, p$ and $f: C \times C \rightarrow \mathcal{R}$ satisfy the following conditions:

(i) $f(x, x)=0$ for all $x \in C$, $f$ is pseudomonotone on $C$,

(ii) $f$ is continuous on $C$,

(iii) For each $x \in C, f(x, \cdot)$ is convex and subdifferentiable on $C$,

(iv) If the sequence $\left\{t^{n}\right\}$ is bounded then $\left\{v^{n}\right\}$ is also bounded, where $v^{n} \in \partial_{2} f\left(t^{n}, t^{n}\right)$,

(v) $\cap_{i=1}^{p} \operatorname{Fix}\left(S_{i}\right) \cap \operatorname{Sol}(f, C) \neq \emptyset$.

Then the sequences $\left\{x^{n}\right\},\left\{y^{n}\right\}$ and $\left\{w^{n}\right\}$ generated by Algorithm 2.1 converge weakly to the point $x^{*}$, where $x^{*}=\lim _{n \rightarrow \infty} \operatorname{Pr}_{\cap_{i=1}^{p} \operatorname{Fix}\left(S_{i}, C\right) \cap S o l(f, C)}\left(x^{n}\right)$.

Proof. We divide the proof into several steps.

Step 1. If there exists $n_{0}$ such that $x^{n}=y^{n}$ for all $n \geq n_{0}$, then the sequences $\left\{x^{n}\right\},\left\{y^{n}\right\}$ and $\left\{w^{n}\right\}$ generated by Algorithm 2.1 converge weakly to $\bar{x} \in \cap_{i=1}^{p} S_{i} \cap \operatorname{Sol}(f, C)$.

Indeed, since $x^{n}=y^{n}$ for all $n \geq n_{0}$, we have $w^{n}=x^{n}$ and

$$
x^{n+1}=\alpha_{n} x^{n}+\left(1-\alpha_{n}\right) \sum_{i=1}^{p} S_{i}\left(x^{n}\right), \forall n \geq n_{0} .
$$

This iteration process is originally introduced by Marino and $\mathrm{Xu}$ in a real Hilbert space (see [5]). Under assumptions of Algorithm 2.1 on the sequence $\left\{\lambda_{n, i}\right\}$, the author showed that the sequence $\left\{x^{n}\right\}$ converges weakly to the same point $\bar{x} \in \cap_{i=1}^{p} \operatorname{Fix}\left(S_{i}\right)$. Then, the sequence $\left\{x^{n}\right\}$ converges weakly to $\bar{x} \in \cap_{i=1}^{p} S_{i} \cap \operatorname{Sol}(f, C)$ in $\mathcal{H}$. Consequently, the sequences $\left\{y^{n}\right\}$ and $\left\{w^{n}\right\}$ also converge weakly to $\bar{x}$ as $n \rightarrow \propto$. In this case, the sequences $\left\{z^{n}\right\}$ and $\left\{v^{n}\right\}$ might not converge weakly to the point $\bar{x}$.

Otherwise, we consider the following steps.

Step 2. If $\left\|r\left(x^{n}\right)\right\| \neq 0$, then there exists the smallest nonnegative integer $m_{n}$ such that

$$
f\left(x^{n}-\gamma^{m_{n}} r\left(x^{n}\right), \gamma^{n}\right) \leq-\sigma\left\|r\left(x^{n}\right)\right\|^{2} .
$$

For $\left\|r\left(x^{n}\right)\right\| \neq 0$ and $\gamma \in(0,1)$, we suppose for contradiction that for every nonnegative integer $m$, we have

$$
f\left(x^{n}-\gamma^{m} r\left(x^{n}\right), y^{n}\right)+\sigma\left\|r\left(x^{n}\right)\right\|^{2}>0 .
$$

Passing to the limit above inequality as $m \rightarrow \propto$, by continuity of $f$, we obtain

$$
f\left(x^{n}, y^{n}\right)+\sigma\left\|r\left(x^{n}\right)\right\|^{2} \geq 0 .
$$

On the other hand, since $y^{n}$ is the unique solution of the strongly convex problem

$$
\min \left\{f\left(x^{n}, y\right)+\frac{\beta}{2}\left\|y-x^{n}\right\|^{2}: y \in C\right\},
$$


we have

$$
f\left(x^{n}, y\right)+\frac{\beta}{2}\left\|y-x^{n}\right\|^{2} \geq f\left(x^{n}, y^{n}\right)+\frac{\beta}{2}\left\|y^{n}-x^{n}\right\|^{2}, \forall y \in C .
$$

With $y=x^{n}$, the last inequality implies

$$
f\left(x^{n}, y^{n}\right)+\frac{\beta}{2}\left\|r\left(x^{n}\right)\right\|^{2} \leq 0 .
$$

Combining (3.1) with (3.2), we obtain

$$
\sigma\left\|r\left(x^{n}\right)\right\|^{2} \geq \frac{\beta}{2}\left\|r\left(x^{n}\right)\right\|^{2} .
$$

Hence it must be either $\left\|r\left(x^{n}\right)\right\|=0$ or $\sigma \geq \frac{\beta}{2}$. The first case contradicts to $\left\|r\left(x^{n}\right)\right\|$ $\neq 0$, while the second one contradicts to the fact $\sigma<\frac{\beta}{2}$.

Step 3. We claim that if $\left\|r\left(x^{n}\right)\right\| \neq 0$ then $x^{n} \notin H_{n}$.

From $z^{n}=x^{n}-\gamma^{m_{n}} r\left(x^{n}\right)$, it follows that

$$
\gamma^{n}-z^{n}=\frac{1-\gamma^{m_{n}}}{\gamma^{m_{n}}}\left(z^{n}-x^{n}\right) .
$$

Then using (4.1) and the assumption $f(x, x)=0$ for all $x \in C$, we have

$$
\begin{aligned}
0 & >-\sigma\left\|r\left(x^{n}\right)\right\|^{2} \\
& \geq f\left(z^{n}, y^{n}\right) \\
& =f\left(z^{n}, y^{n}\right)-f\left(z^{n}, z^{n}\right) \\
& \geq\left\langle v^{n}, y^{n}-z^{n}\right\rangle \\
& =\frac{1-\gamma^{m_{n}}}{\gamma^{m_{n}}}\left\langle z^{n}-x^{n}, v^{n}\right\rangle .
\end{aligned}
$$

Hence

$$
\left\langle x^{n}-z^{n}, v^{n}\right\rangle>0 .
$$

This implies that $x^{n} \notin H_{n}$.

Step 4. We claim that if $\left\|r\left(x^{n}\right)\right\| \neq 0$ then $w^{n}=\operatorname{Pr}_{C \cap H_{n}}\left(\bar{y}^{n}\right)$, where $\bar{y}^{n}=\operatorname{Pr}_{H_{n}}\left(x^{n}\right)$.

For $K=\left\{x \in \mathcal{H}:\left\langle w, x-x^{0}\right\rangle \leq 0\right\}$ and $\|w\| \neq 0$, we know that

$$
\operatorname{Pr}_{K}(y)=y-\frac{\left\langle w, y-x^{0}\right\rangle}{\|w\|^{2}} w
$$

Hence,

$$
\begin{aligned}
\bar{y}^{n} & =\operatorname{Pr}_{H_{n}}\left(x^{n}\right) \\
& =x^{n}-\frac{\left\langle v^{n}, x^{n}-z^{n}\right\rangle}{\left\|v^{n}\right\|^{2}} v^{n} \\
& =x^{n}-\frac{\gamma^{m_{n}}\left\langle v^{n}, r\left(x^{n}\right)\right\rangle}{\left\|v^{n}\right\|^{2}} v^{n} .
\end{aligned}
$$

Otherwise, for every $y \in C \cap H_{n}$ there exists $\lambda \in(0,1)$ such that

$$
\hat{x}=\lambda x^{n}+(1-\lambda) y \in C \cap \partial H_{n}
$$


where

$$
\partial H_{n}=\left\{x \in \mathcal{H}:\left\langle v^{n}, x-z^{n}\right\rangle=0\right\} .
$$

From Step 2, it follows that $x^{n} \in C$ but $x^{n} \notin H_{n}$. Therefore, we have

$$
\begin{aligned}
\left\|y-\bar{y}^{n}\right\|^{2} & \geq(1-\lambda)^{2}\left\|y-\bar{y}^{n}\right\|^{2} \\
& =\left\|\hat{x}-\lambda x^{n}-(1-\lambda) \bar{y}^{n}\right\|^{2} \\
& =\left\|\left(\hat{x}-\bar{y}^{n}\right)-\lambda\left(x^{n}-\bar{y}^{n}\right)\right\|^{2} \\
& =\left\|\hat{x}-\bar{y}^{n}\right\|^{2}+\lambda^{2}\left\|x^{n}-\bar{y}^{n}\right\|^{2}-2 \lambda\left\langle\hat{x}-\bar{y}^{n}, x^{n}-\bar{y}^{n}\right\rangle \\
& =\left\|\hat{x}-\bar{y}^{n}\right\|^{2}+\lambda^{2}\left\|x^{n}-\bar{y}^{n}\right\|^{2} \\
& \geq\left\|\hat{x}-\bar{y}^{n}\right\|^{2},
\end{aligned}
$$

because $\bar{y}^{n}=\operatorname{Pr}_{H_{n}}\left(x^{n}\right)$. Also we have

$$
\begin{aligned}
\left\|\hat{x}-x^{n}\right\|^{2} & =\left\|\hat{x}-\bar{y}^{n}+\bar{y}^{n}-x^{n}\right\|^{2} \\
& =\left\|\hat{x}-\bar{y}^{n}\right\|^{2}-2\left\langle\hat{x}-\bar{y}^{n}, x^{n}-\bar{y}^{n}\right\rangle+\left\|\bar{y}^{n}-x^{n}\right\|^{2} \\
& =\left\|\hat{x}-\bar{y}^{n}\right\|^{2}+\left\|\bar{y}^{n}-x^{n}\right\|^{2} .
\end{aligned}
$$

Using $w^{n}=\operatorname{Pr}_{C \cap H_{n}}\left(x^{n}\right)$ and the Pythagorean theorem, we can reduce that

$$
\begin{aligned}
\left\|\hat{x}-\bar{y}^{n}\right\|^{2} & =\left\|\hat{x}-x^{n}\right\|^{2}-\left\|\bar{y}^{n}-x^{n}\right\|^{2} \\
& \geq\left\|w^{n}-x^{n}\right\|^{2}-\left\|\bar{y}^{n}-x^{n}\right\|^{2} \\
& =\left\|w^{n}-\bar{y}^{n}\right\|^{2} .
\end{aligned}
$$

From (3.3) and (3.4), we have

$$
\left\|w^{n}-\bar{y}^{n}\right\| \leq\left\|y-\bar{\gamma}^{n}\right\|, \quad \forall y \in C \cap H_{n}
$$

which means

$$
w^{n}=\operatorname{Pr}_{C \cap H_{n}}\left(\bar{y}^{n}\right) .
$$

Step 5. We claim that if $\left\|r\left(x^{n}\right)\right\| \neq 0$ then $\operatorname{Sol}(f, C) \subseteq C \cap H_{n}$.

Indeed, suppose $x^{*} \in \operatorname{Sol}(f, C)$. Using the definition of $x^{*}, f\left(x^{* *}, x\right) \geq 0$ for all $x \in C$ and $f$ is pseudomonotone on $C$, we get

$$
f\left(z^{n}, x^{*}\right) \leq 0 .
$$

It follows from $v^{n} \in \partial_{2} f\left(z^{n}, z^{n}\right)$ that

$$
\begin{aligned}
f\left(z^{n}, x^{*}\right) & =f\left(z^{n}, x^{*}\right)-f\left(z^{n}, z^{n}\right) \\
& \geq\left\langle v^{n}, x^{*}-z^{n}\right\rangle .
\end{aligned}
$$

Combining (3.5) and (3.6), we have

$$
\left\langle v^{n}, x^{*}-z^{n}\right\rangle \leq 0 .
$$

By the definition of $H_{n}$, we have $x^{*} \in H_{n}$. Thus $\operatorname{Sol}(f, C) \subseteq C \cap H_{n}$.

Step 6. We claim that if $\left\|r\left(x^{n}\right)\right\| \neq 0$ and the sequence $\left\{v^{n}\right\}$ is uniformly bounded by $M>0$ then the sequence $\left\{\| x^{n}-x^{*}||\right\}$ is nonincreasing and hence convergent. Moreover, we have

$$
\begin{gathered}
\left\|x^{n+1}-x^{*}\right\|^{2} \leq\left\|x^{n}-x^{*}\right\|^{2}-\left(1-\alpha_{n}\right)\left\|w^{n}-\bar{y}^{n}\right\|^{2}-\left(1-\alpha_{n}\right)\left(\frac{\gamma^{m_{n}} \sigma}{M\left(1-\gamma^{m_{n}}\right)}\right)^{2}\left\|r\left(x^{n}\right)\right\|^{4} \\
-\left(1-\alpha_{n}\right)\left(\alpha_{n}-\bar{L}\right)\left\|\bar{S}_{n}\left(w^{n}\right)-w^{n}\right\|^{2},
\end{gathered}
$$


where $\bar{y}^{n}=\operatorname{Pr}_{H_{n}}\left(x^{n}\right), \bar{S}_{n}:=\sum_{i=1}^{p} \lambda_{n, i} S_{i}$ and $x^{*} \in \bigcap_{i=1}^{p} \operatorname{Fix}\left(S_{i}\right) \cap \operatorname{Sol}(f, C)$.

In the case $\left\|r\left(x^{n}\right)\right\| \neq 0$, by Step 4 , we have $w^{n}=\operatorname{Pr}_{C \cap H_{n}}\left(\bar{y}^{n}\right)$, i.e.,

$$
\left\langle\bar{y}^{n}-w^{n}, z-w^{n}\right\rangle \leq 0, \forall z \in C \cap H_{n}
$$

where $\bar{y}^{n}=\operatorname{Pr}_{H_{n}}\left(x^{n}\right)$. Substituting $z=x^{*} \in \operatorname{Sol}(f, C) \subseteq C \cap H_{n}$ by Step 5, then we have

$$
\left\langle\bar{y}^{n}-w^{n}, x^{*}-w^{n}\right\rangle \leq 0 \Leftrightarrow\left\langle y^{n}-w^{n}, x^{*}-\bar{y}^{n}+\bar{y}^{n}-w^{n}\right\rangle \leq 0,
$$

which implies that

$$
\left\|w^{n}-\bar{y}^{n}\right\|^{2} \leq\left\langle w^{n}-\bar{y}^{n}, x^{*}-\bar{y}^{n}\right\rangle .
$$

Hence

$$
\begin{aligned}
\left\|w^{n}-x^{*}\right\|^{2} & =\left\|w^{n}-\bar{y}^{n}+\bar{y}^{n}-x^{*}\right\|^{2} \\
& =\left\|w^{n}-\bar{y}^{n}\right\|^{2}+\left\|\bar{y}^{n}-x^{*}\right\|^{2}+2\left\langle w^{n}-\bar{y}^{n}, \bar{y}^{n}-x^{*}\right\rangle \\
& \leq\left\langle x^{*}-\bar{y}^{n}, w^{n}-\bar{y}^{n}\right\rangle+\left\|\bar{y}^{n}-x^{*}\right\|^{2}+2\left\langle w^{n}-\bar{y}^{n}, \bar{y}^{n}-x^{*}\right\rangle \\
& =\left\|\bar{y}^{n}-x^{*}\right\|^{2}+\left\langle w^{n}-\bar{y}^{n}, \bar{y}^{n}-x^{*}\right\rangle \\
& \leq\left\|\bar{y}^{n}-x^{*}\right\|^{2}-\left\|w^{n}-\bar{y}^{n}\right\|^{2} .
\end{aligned}
$$

Since $z^{n}=x^{n}-\gamma^{m_{n}} r\left(x^{n}\right)$ and

$$
\bar{y}^{n}=\operatorname{Pr}_{H_{n}}\left(x^{n}\right)=x^{n}-\frac{\left\langle v^{n}, x^{n}-z^{n}\right\rangle}{\left\|v^{n}\right\|^{2}} v^{n},
$$

we have

$$
\begin{aligned}
\| \bar{y}^{n}- & x^{*} \|^{2} \\
= & \left\|x^{n}-x^{*}\right\|^{2}+\frac{\left\langle v^{n}, x^{n}-z^{n}\right\rangle^{2}}{\left\|v^{n}\right\|^{4}}\left\|v^{n}\right\|^{2}-\frac{2\left\langle v^{n}, x^{n}-z^{n}\right\rangle}{\left\|v^{n}\right\|^{2}}\left\langle v^{n}, x^{n}-x^{*}\right\rangle \\
= & \left\|x^{n}-x^{*}\right\|^{2}+\left(\frac{\gamma^{m_{n}}\left\langle v^{n}, r\left(x^{n}\right)\right\rangle}{\left\|v^{n}\right\|}\right)^{2}-\frac{2 \gamma^{m_{n}}\left\langle v^{n}, r\left(x^{n}\right)\right\rangle}{\left\|v^{n}\right\|^{2}}\left\langle v^{n}, x^{n}-x^{*}\right\rangle \\
= & \left\|x^{n}-x^{*}\right\|^{2}+\left(\frac{\gamma^{m_{n}}\left\langle v^{n}, r\left(x^{n}\right)\right\rangle}{\left\|v^{n}\right\|}\right)^{2} \\
& -2\left(\frac{\gamma^{m_{n}}\left\langle v^{n}, r\left(x^{n}\right)\right\rangle}{\left\|v^{n}\right\|^{2}}\left\langle v^{n}, x^{n}-x^{*}\right\rangle-\left(\frac{\gamma^{m_{n}}\left\langle v^{n}, r\left(x^{n}\right)\right\rangle}{\left\|v^{n}\right\|}\right)^{2}\right) \\
= & \left\|x^{k}-x^{*}\right\|^{2}-\left(\frac{\gamma^{m_{n}}\left\langle v^{n}, r\left(x^{n}\right)\right\rangle}{\left\|v^{n}\right\|}\right)^{2} \\
& -\frac{2 \gamma^{m_{n}}\left\langle v^{n}, r\left(x^{n}\right)\right\rangle}{\left\|v^{n}\right\|^{2}}\left(\left\langle v^{n}, x^{n}-x^{*}\right\rangle-\gamma^{m_{n}}\left\langle v^{n}, r\left(x^{n}\right)\right\rangle\right) \\
= & \left\|x^{n}-x^{*}\right\|^{2}-\left(\frac{\gamma^{m_{n}}\left\langle v^{n}, r\left(x^{n}\right)\right\rangle}{\left\|v^{n}\right\|}\right)^{2} \\
& -\frac{2 \gamma^{m_{n}}\left\langle v^{n}, r\left(x^{n}\right)\right\rangle}{\left\|v^{n}\right\|^{2}}\left\langle v^{n}, x^{n}-x^{*}-\gamma^{m_{n}} r\left(x^{n}\right)\right\rangle \\
= & \left\|x^{n}-x^{*}\right\|^{2}-\left(\frac{\gamma^{m_{n}}\left\langle v^{n}, r\left(x^{n}\right)\right\rangle}{\left\|v^{n}\right\|}\right)^{2}-\frac{2 \gamma^{m_{n}}\left\langle v^{n}, r\left(x^{n}\right)\right\rangle}{\left\|v^{n}\right\|^{2}}\left\langle v^{n}, z^{n}-x^{*}\right\rangle .
\end{aligned}
$$

It follows from $v^{n} \in \partial_{2} f\left(z^{n}, z^{n}\right)$ that

$$
f\left(z^{n}, y\right)-f\left(z^{n}, z^{n}\right) \geq\left\langle v^{n}, y-z^{n}\right\rangle, \quad \forall y \in C .
$$


Ant and Hen Fixed Point Theory and Applications 2012, 2012:82

Page 9 of 16

http://www.fixedpointtheoryandapplications.com/content/2012/1/82

Replacing $y$ by $y^{n}$ and combining with assumptions $f\left(z^{n}, z^{n}\right)=0$ and $z^{n}=x^{n}-\gamma^{m_{n}} r\left(x^{n}\right)$

we have

$$
\begin{aligned}
f\left(z^{n}, \gamma^{n}\right) & \geq\left\langle v^{n}, y^{n}-z^{n}\right\rangle \\
& =-\left(1-\gamma^{m_{n}}\right)\left\langle v^{n}, r\left(x^{n}\right)\right\rangle .
\end{aligned}
$$

Combining this inequality with (4.1) and assumption $\gamma \in(0,1)$, we obtain

$$
\left\langle v^{n}, r\left(x^{n}\right)\right\rangle \geq \frac{\sigma}{1-\gamma^{m_{n}}}\left\|r\left(x^{n}\right)\right\|^{2} .
$$

Substituting $y=x^{*}$ into (3.10) and using $f\left(z^{n}, z^{n}\right)=0$, we have

$$
f\left(z^{n}, x^{*}\right) \geq\left\langle v^{n}, x^{*}-z^{n}\right\rangle .
$$

Since $f$ is pseudomonotone on $C$ and $f\left(x^{*}, x\right) \geq 0, \forall x \in C$, we have

$$
f\left(z^{n}, x^{*}\right) \leq 0
$$

Combining this with (3.12), we get

$$
0 \geq\left\langle v^{n}, x^{*}-z^{n}\right\rangle
$$

Using (3.9), (3.11) and (3.13), we have

$$
\begin{aligned}
\left\|\bar{y}^{n}-x^{*}\right\|^{2} & \leq\left\|x^{n}-x^{*}\right\|^{2}-\left(\frac{\gamma^{m_{n}}\left\langle v^{n}, r\left(x^{n}\right)\right\rangle}{\left\|v^{n}\right\|}\right)^{2} \\
& \leq\left\|x^{n}-x^{*}\right\|^{2}-\left(\frac{\gamma^{m_{n}} \sigma}{\left\|v^{n}\right\|\left(1-\gamma^{m_{n}}\right)}\right)^{2}\left\|r\left(x^{n}\right)\right\|^{4} .
\end{aligned}
$$

Combining (3.8) with (3.14), we obtain

$$
\left\|w^{n}-x^{*}\right\|^{2} \leq\left\|x^{n}-x^{*}\right\|^{2}-\left\|w^{n}-\bar{y}^{n}\right\|^{2}-\left(\frac{\gamma^{m_{n}} \sigma}{\left\|v^{n}\right\|\left(1-\gamma^{m_{n}}\right)}\right)^{2}\left\|r\left(x^{n}\right)\right\|^{4} .
$$

Using $\bar{S}_{n}:=\sum_{i=1}^{p} \lambda_{n, i} S_{i},(3.15), x^{n+1}=\alpha_{n} w^{n}+\left(1-\alpha_{n}\right) \sum_{i=1}^{p} \lambda_{n, i} S_{i}\left(w^{n}\right)$ and the equality

$$
\|\lambda x+(1-\lambda) y\|^{2}=\lambda\|x\|^{2}+(1-\lambda)\|y\|^{2}-\lambda(1-\lambda)\|x-y\|^{2}, \forall \lambda \in[0,1], x, y \in \mathcal{R}^{n},
$$

we have

$$
\begin{aligned}
&\left\|x^{n+1}-x^{*}\right\|^{2} \\
&=\left\|\alpha_{n} w^{n}+\left(1-\alpha_{n}\right) \bar{S}_{n}\left(w^{n}\right)-x^{*}\right\|^{2} \\
&=\left\|\alpha_{n}\left(w^{n}-x^{*}\right)+\left(1-\alpha_{n}\right)\left(\bar{S}_{n}\left(w^{n}\right)-x^{*}\right)\right\|^{2} \\
&= \alpha_{n}\left\|w^{n}-x^{*}\right\|^{2}+\left(1-\alpha_{n}\right)\left\|\bar{S}_{n}\left(w^{n}\right)-\bar{S}_{n}\left(x^{*}\right)\right\|^{2}-\alpha_{n}\left(1-\alpha_{n}\right)\left\|\bar{S}_{n}\left(w^{n}\right)-w^{n}\right\|^{2} \\
& \leq \alpha_{n}\left\|w^{n}-x^{*}\right\|^{2}+\left(1-\alpha_{n}\right)\left(\left\|w^{n}-x^{*}\right\|^{2}+\bar{L}\left\|\left(I-\bar{S}_{n}\right)\left(w^{n}\right)-\left(I-\bar{S}_{n}\right)\left(x^{*}\right)\right\|^{2}\right) \\
&-\alpha_{n}\left(1-\alpha_{n}\right)\left\|\bar{S}_{n}\left(w^{n}\right)-w^{n}\right\|^{2} \\
&=\left\|w^{n}-x^{*}\right\|^{2}+\left(1-\alpha_{n}\right)\left(\bar{L}-\alpha_{n}\right)\left\|\bar{S}_{n}\left(w^{n}\right)-w^{n}\right\|^{2} \\
& \leq\left\|x^{n}-x^{*}\right\|^{2}-\left(1-\alpha_{n}\right)\left\|w^{n}-\bar{\gamma}^{n}\right\|^{2}-\left(1-\alpha_{n}\right)\left(\frac{\gamma^{m_{n}} \sigma}{\left\|v^{n}\right\|\left(1-\gamma^{m_{n}}\right)}\right)^{2}\left\|r\left(x^{n}\right)\right\|^{4} \\
&-\left(1-\alpha_{n}\right)\left(\alpha_{n}-\bar{L}\right)\left\|\bar{S}_{n}\left(w^{n}\right)-w^{n}\right\|^{2} .
\end{aligned}
$$


In the case $\left\|r\left(x^{n}\right)\right\|=0$, by Algorithm 2.1 and (3.16), we have $w^{n}=x^{n}$ and

$$
\begin{aligned}
\left\|x^{n+1}-x^{*}\right\|^{2}= & \left\|\alpha_{n} x^{n}+\left(1-\alpha_{n}\right) \bar{S}_{n}\left(x^{n}\right)-x^{*}\right\|^{2} \\
= & \left\|\alpha_{n}\left(x^{n}-x^{*}\right)+\left(1-\alpha_{n}\right)\left(\bar{S}_{n}\left(x^{n}\right)-\bar{S}_{n}\left(x^{*}\right)\right)\right\|^{2} \\
= & \alpha_{n}\left\|x^{n}-x^{*}\right\|^{2}+\left(1-\alpha_{n}\right)\left\|\bar{S}_{n}\left(x^{n}\right)-\bar{S}_{n}\left(x^{*}\right)\right\|^{2} \\
& -\alpha_{n}\left(1-\alpha_{n}\right)\left\|\left(I-\bar{S}_{n}\right)\left(x^{n}\right)-\left(I-\bar{S}_{n}\right)\left(x^{*}\right)\right\|^{2} \\
\leq & \alpha_{n}\left\|x^{n}-x^{*}\right\|^{2}+\left(1-\alpha_{n}\right)\left(\left\|x^{n}-x^{*}\right\|^{2}+\bar{L}\left\|\left(I-\bar{S}_{n}\right)\left(x^{n}\right)-\left(I-\bar{S}_{n}\right)\left(x^{*}\right)\right\|^{2}\right) \\
& -\alpha_{n}\left(1-\alpha_{n}\right)\left\|\left(I-\bar{S}_{n}\right)\left(x^{n}\right)-\left(I-\bar{S}_{n}\right)\left(x^{*}\right)\right\|^{2} \\
= & \left\|x^{n}-x^{*}\right\|-\left(1-\alpha_{n}\right)\left(\alpha_{n}-\bar{L}\right)\left\|\bar{S}_{n}\left(x^{n}\right)-x^{n}\right\| \\
\leq & \left\|x^{n}-x^{*}\right\| .
\end{aligned}
$$

Combining this and (3.17), we get

$$
\left\|x^{n+1}-x^{*}\right\| \leq\left\|x^{n}-x^{*}\right\|, \quad \forall n \geq 0 .
$$

So the sequence $\left\{\left\|x^{n}-x^{*}\right\|\right\}$ is nonincreasing and hence convergent. Since (3.17) and the sequence $\left\{v^{n}\right\}$ is uniformly bounded by $M>0$, i.e.,

$$
\left\|v^{n}\right\| \leq M, \forall n \geq 0,
$$

we obtain (3.7).

Step 7. We claim that there exists $c=\lim _{n \rightarrow \infty}\left\|x^{n}-x^{*}\right\|=\lim _{n \rightarrow \infty}\left\|w^{n}-x^{*}\right\|$, where $x^{*} \in \cap_{i=1}^{p} \operatorname{Fix}\left(S_{i}\right) \cap \operatorname{Sol}(f, C)$. Consequently, the sequences $\left\{x^{n}\right\},\left\{y^{n}\right\},\left\{z^{n}\right\},\left\{v^{n}\right\}$ and $\left\{w^{n}\right\}$ are bounded.

By Step 6, there exists

$$
c=\lim _{n \rightarrow \infty}\left\|x^{n}-x^{*}\right\| .
$$

From $w^{n}=x^{n}$ if $\left\|r\left(x^{n}\right)\right\|=0, w^{n}=\operatorname{Pr}_{C \cap H_{n}}\left(x^{n}\right)$ if $\left\|r\left(x^{n}\right)\right\| \neq 0$ and Step 6, it follows that

$$
\left\|w^{n}-x^{*}\right\| \leq\left\|x^{n}-x^{*}\right\|, \forall n \geq 0 .
$$

Hence

$$
\lim _{n \rightarrow \infty}\left\|w^{n}-x^{*}\right\| \leq \lim _{n \rightarrow \infty}\left\|x^{n}-x^{*}\right\|=c .
$$

Using $x^{n+1}=\alpha_{n} w^{n}+\left(1-\alpha_{n}\right) \bar{S}_{n}\left(w^{n}\right)$, we have

$$
\begin{aligned}
\left\|x^{n+1}-x^{*}\right\|^{2}= & \left\|\alpha_{n} w^{n}+\left(1-\alpha_{n}\right) \bar{S}_{n}\left(w^{n}\right)-x^{*}\right\|^{2} \\
= & \left\|\alpha_{n}\left(w^{n}-x^{*}\right)+\left(1-\alpha_{n}\right)\left(\bar{S}_{n}\left(w^{n}\right)-x^{*}\right)\right\|^{2} \\
= & \alpha_{n}\left\|w^{n}-x^{*}\right\|^{2}+\left(1-\alpha_{n}\right)\left\|\bar{S}_{n}\left(w^{n}\right)-\bar{S}_{n}\left(x^{*}\right)\right\|^{2} \\
& -\alpha_{n}\left(1-\alpha_{n}\right)\left\|\left(I-\bar{S}_{n}\right)\left(w^{n}\right)-\left(I-\bar{S}_{n}\right)\left(x^{*}\right)\right\|^{2} \\
\leq & \alpha_{n}\left\|w^{n}-x^{*}\right\|^{2}+\left(1-\alpha_{n}\right)\left(\left\|w^{n}-x^{*}\right\|^{2}+\bar{L}\left\|\bar{S}_{n}\left(w^{n}\right)-w^{n}\right\|^{2}\right) \\
& -\alpha_{n}\left(1-\alpha_{n}\right)\left\|\left(I-\bar{S}_{n}\right)\left(w^{n}\right)-\left(I-\bar{S}_{n}\right)\left(x^{*}\right)\right\|^{2} \\
= & \left\|w^{n}-x^{*}\right\|^{2}-\left(1-\alpha_{n}\right)\left(\alpha_{n}-\bar{L}\right)\left\|\bar{S}_{n}\left(w^{n}\right)-w^{n}\right\|^{2} \\
\leq & \left\|w^{n}-x^{*}\right\| .
\end{aligned}
$$


Hence

$$
c \leq \lim _{n \rightarrow \infty}\left\|w^{n}-x^{*}\right\| .
$$

From (3.21) and (3.19), it follows that

$$
c=\lim _{n \rightarrow \infty}\left\|w^{n}-x^{*}\right\| .
$$

Since $y^{n}$ is the unique solution to

$$
\min \left\{f\left(x^{n}, y\right)+\frac{\beta}{2}\left\|y-x^{n}\right\|^{2}: y \in C\right\}
$$

we have

$$
f\left(x^{n}, y\right)+\frac{\beta}{2}\left\|y-x^{n}\right\|^{2} \geq f\left(x^{n}, y^{n}\right)+\frac{\beta}{2}\left\|y^{n}-x^{n}\right\|^{2}, \quad \forall y \in C .
$$

With $y=x^{n} \in C$ and $f\left(x^{n}, x^{n}\right)=0$, we have

$$
0 \geq f\left(x^{n}, y^{n}\right)+\frac{\beta}{2}\left\|y^{n}-x^{n}\right\|^{2} .
$$

Since $f\left(x^{n}, \cdot\right)$ is convex and subdifferentiable on $C$, i.e.,

$$
f\left(x^{n}, y\right)-f\left(x^{n}, x^{n}\right) \geq\left\langle u^{n}, y-x^{n}\right\rangle \quad \forall y \in C,
$$

where $u^{n} \in \partial_{2} f\left(x^{n}, x^{n}\right)$. Using $y=y^{n}$, we have

$$
f\left(x^{n}, y^{n}\right) \geq\left\langle u^{n}, y^{n}-x^{n}\right\rangle .
$$

Combining this and (3.22), we obtain

$$
\left\langle u^{n}, y^{n}-x^{n}\right\rangle+\frac{\beta}{2}\left\|x^{n}-y^{n}\right\|^{2} \leq 0 .
$$

Hence

$$
\left\|x^{n}-y^{n}+\frac{1}{\beta} u^{n}\right\| \leq \frac{1}{\beta}\left\|u^{n}\right\| .
$$

From the assumption (iv) and (3.18), it implies that the sequence $\left\{u^{n}\right\}$ is bounded. Then, it follows from (3.23) that $\left\{y^{n}\right\}$ is bounded and hence $z^{n}=x^{n}-\gamma^{m_{n}}\left(x^{n}-\gamma^{n}\right)$ is also bounded. Also the sequences $\left\{v^{n}\right\}$ and $\left\{w^{n}\right\}$ are bounded.

Step 8. We claim that there exists a subsequence of the sequence $\left\{x^{n}\right\}$ which converges weakly to $\bar{x} \in \cap_{i=1}^{p}$ Fix $\left(S_{i}\right)$ Sol $(f, C)$ and hence the whole sequence $\left\{x^{n}\right\}$ converges weakly to $\bar{x}$.

Suppose that $\left\{x^{n_{k}}\right\}$ is a subsequence of $\left\{x^{n}\right\}$ such that

$$
\left\|r\left(x^{n_{k}}\right)\right\| \neq 0 .
$$

By Step 7 and the assumption (iv), the sequence $\left\{v^{n}\right\}$ is bounded by $M>0$. We show that 


$$
\begin{aligned}
\left\|x^{n_{k+1}}-x^{*}\right\|^{2} \leq\left\|x^{n_{k}}-x^{*}\right\|^{2} & -(1-b)\left\|w^{n_{k}+p}-\bar{y}^{n_{k}+p}\right\|^{2} \\
& -b(a-\bar{L})\left\|\bar{S}_{n_{k}+p}\left(w^{n_{k}+p}\right)-w^{n_{k}+p}\right\|^{2} \\
& -(1-b)\left(\frac{\gamma^{m_{n_{k}+p}} \sigma}{M\left(1-\gamma^{m_{n_{k}+p}}\right)}\right)^{2}\left\|r\left(x^{n_{k}+p}\right)\right\|^{4},
\end{aligned}
$$

where $\quad p=n_{k+1}-n_{k}-1, \bar{y}^{n_{k}+p}=\operatorname{Pr}_{H_{n_{k}+p}}\left(x^{n_{k}+p}\right), \quad x^{*} \in \cap_{i=1}^{p} \operatorname{Fix}\left(S_{i}\right) \cap \operatorname{Sol}(f, C) \quad$ and $\bar{S}_{n}:=\sum_{i=1}^{p} \lambda_{n, i} S_{i}$. Indeed, if $n_{k+1}=n_{k}+1$ then it is clear from Step 6. Otherwise, we suppose that there exists a positive integer $p$ such that $n_{k}+p+1=n_{k+1}$. Note that $\left\|r\left(x^{n_{k}+i}\right)\right\|=0$ for all $i=0,1, \ldots, p-1$. Using $r\left(x^{n_{k}+p}\right) \neq 0$, (3.17) and Step 6, we have

$$
\begin{aligned}
\left\|x^{n_{k+1}}-x^{*}\right\|^{2}= & \left\|x^{n_{k}+p+1}-x^{*}\right\|^{2} \\
\leq & \left\|x^{n_{k}+p}-x^{*}\right\|^{2}-\left(1-\alpha_{n_{k}+p}\right)\left\|w^{n_{k}+p}-\bar{\gamma}^{n_{k}+p}\right\|^{2} \\
& -\left(1-\alpha_{n_{k}+p}\right)\left(\frac{\gamma^{m_{n_{k}+p}} \sigma}{\left\|v^{n_{k}+p}\right\|\left(1-\gamma^{m_{n_{k}+p}}\right)}\right)^{2}\left\|r\left(x^{n_{k}+p}\right)\right\|^{4} \\
& -\left(1-\alpha_{n_{k}+p}\right)\left(\alpha_{n_{k}+p}-\bar{L}\right)\left\|\bar{S}_{n_{k}+p}\left(w^{n_{k}+p}\right)-w^{n_{k}+p}\right\|^{2} \\
\leq & \cdots \\
\leq & \left\|x^{n_{k}}-x^{*}\right\|^{2}-(1-b)\left\|w^{n_{k}+p}-\bar{y}_{n_{k}+p}\right\|^{2} \\
& -b(a-\bar{L})\left\|\bar{S}_{n_{k}+p}\left(w^{n_{k}+p}\right)-w^{n_{k}+p}\right\|^{2} \\
& -(1-b)\left(\frac{\gamma^{m_{n_{k}+p}} \sigma}{M\left(1-\gamma^{m_{n_{k}+p}}\right)}\right)^{2}\left\|r\left(x^{n_{k}+p}\right)\right\|^{4} .
\end{aligned}
$$

This implies (3.24). Then, since $\left\{\left\|x^{n}-x^{*}\right\|\right\}$ is convergent, it is easy to see that

$$
\lim _{k \rightarrow \infty} \gamma^{m_{n_{k}+p}}\left\|r\left(x^{n_{k}+p}\right)\right\|=0 .
$$

The cases remaining to consider are the following.

Case 1. $\limsup _{k \rightarrow \infty} \gamma^{m_{n_{k}+p}}>0$. This case must follow that $\liminf _{k \rightarrow \infty}\left\|r\left(x^{n_{k}+p}\right)\right\|=0$. Since $\left\{x^{n_{k}+p}\right\}$ is bounded, there exists an accumulation point $\bar{x}$ of $\left\{x^{n_{k}+p}\right\}$. In other words, a subsequence $\left\{x^{n_{k_{j}}}\right\}$ converges weakly to some $\bar{x}$, as $j \rightarrow \propto$ such that $r(\bar{x})=0$. Then by Remark 2.2, we have $\bar{x} \in \operatorname{Sol}(f, C)$.

Case 2. $\lim _{k \rightarrow \infty} \gamma^{m_{n_{k}+p}}=0$. Since $\left\{|| x^{n_{k}+p}-x^{*} \|\right\}$ is convergent, there is the subsequence $\left\{x^{n_{k}+p}\right\}$ of $\left\{x^{n_{k}+p}\right\}$ which converges weakly to $\bar{x}$, as $j \rightarrow \propto$. Since $m_{n_{k}+p}$ is the smallest nonnegative integer, $m_{n_{k}+p}-1$ does not satisfy (4.1). Hence, we have

$$
f\left(x^{n_{k_{j}}}-\gamma^{m_{n_{k_{j}}-1}} r\left(x^{n_{k_{j}}}\right), \gamma^{n_{k_{j}}}\right)>-\sigma\left\|r\left(x^{n_{k_{j}}}\right)\right\|^{2} .
$$

Passing onto the limit, as $j \rightarrow \alpha$ and using the continuity of $f$, we have $y^{n_{k_{j}}} \rightarrow \bar{y}$ and

$$
f(\bar{x}, \bar{y}) \geq-\sigma\|r(\bar{x})\|^{2},
$$

where $r(\bar{x})=\bar{x}-\bar{y}$. It follows from (3.2) that

$$
f\left(x^{n_{k_{j}}-1}-\gamma^{m_{n_{k_{j}}}} r\left(x^{n_{k_{j}}}\right), \gamma^{n_{k_{j}}-1}\right)+\frac{\beta}{2}\left\|r\left(x^{n_{k_{j}}-1}\right)\right\|^{2} \leq 0 .
$$

Since $f$ is continuous and passing onto the limit, as $j \rightarrow \alpha$, we obtain

$$
f(\bar{x}, \bar{y})+\frac{\beta}{2}\|r(\bar{x})\|^{2} \leq 0 .
$$


Combining this with (3.25), we have

$$
\sigma\|r(\bar{x})\|^{2} \geq-f(\bar{x}, \bar{y}) \geq \frac{\beta}{2}\|r(\bar{x})\|^{2} .
$$

which implies $r(\bar{x})=0$, and hence $\bar{x}=\bar{y} \in \operatorname{Sol}(f, C)$. Thus every cluster point of the sequence $\left\{x^{n_{k}+p}\right\}$ is a solution to Problem $E P(f, C)$.

Now we show that every cluster point of $\left\{x^{n_{k}+p}\right\}$ is a fixed point of $p$ strict pseudocontractions $\left\{S_{i}\right\}_{i=1}^{p}$. Suppose that there exists a subsequence $\left\{x^{n_{k_{j}}}\right\}$ of $\left\{x^{n_{k}+p}\right\}$ which converges weakly to $\bar{x}$, as $j \rightarrow \propto$. By the above proof, we have $\bar{x} \in \operatorname{Sol}(f, C)$. Then $\left\{\gamma^{n_{k_{j}}}\right\}$ and $\left\{w^{n_{k_{j}}}\right\}$ converge weakly also to $\bar{x}$, as $j \rightarrow \alpha$. For each $i=1, \ldots, p$, we suppose that $\lambda_{n_{k_{j}}, i}$ converges $\lambda_{i}$ as $i \rightarrow \propto$ such that

$$
\sum_{i=1}^{p} \lambda_{i}=1
$$

Then, we have

$$
\bar{S}_{n_{k_{j}}}(x) \rightarrow S(x):=\sum_{i=1}^{p} \lambda_{i} S_{i}(x) \quad(\text { as } j \rightarrow \infty), \quad \forall x \in C .
$$

For each $x^{*} \in \cap_{i=1}^{p} \operatorname{Fix}\left(S_{i}\right) \cap \operatorname{Sol}(f, C)$, it follows from (3.20) that

$$
\left(1-\alpha_{n}\right)\left(\alpha_{n}-\bar{L}\right)\left\|\bar{S}_{n}\left(w^{n}\right)-w^{n}\right\|^{2} \leq\left\|w^{n}-x^{*}\right\|-\left\|x^{n+1}-x^{*}\right\|^{2} .
$$

Combining this and Step 6, we get

$$
\begin{aligned}
\left\|\bar{S}_{n}\left(w^{n}\right)-w^{n}\right\|^{2} & \leq \frac{1}{\left(1-\alpha_{n}\right)\left(\alpha_{n}-\bar{L}\right)}\left(\left\|w^{n}-x^{*}\right\|^{2}-\left\|x^{n+1}-x^{*}\right\|^{2}\right) \\
& \leq \frac{1}{(1-b)(a-\bar{L})}\left(\left\|w^{n}-x^{*}\right\|^{2}-\left\|x^{n+1}-x^{*}\right\|^{2}\right) \\
& \rightarrow 0 \text { as } n \rightarrow \infty .
\end{aligned}
$$

Then, using (a) of Proposition 1.1, we obtain

$$
\begin{aligned}
\left\|x^{n_{k_{j}}}-\bar{S}_{n_{k_{j}}}\left(x^{n_{k_{j}}}\right)\right\| & \leq\left\|x^{n_{k_{j}}}-w^{n_{k_{j}}}\right\|+\left\|w^{n_{k_{j}}}-\bar{S}_{n_{k_{j}}}\left(w^{n_{k_{j}}}\right)\right\|+\left\|\bar{S}_{n_{k_{j}}}\left(w^{n_{k_{j}}}\right)-\bar{S}_{n_{k_{j}}}\left(x^{n_{k_{j}}}\right)\right\| \\
& \leq\left\|x^{n_{k_{j}}}-w^{n_{k_{j}}}\right\|+\left\|w^{n_{k_{j}}}-\bar{S}_{n_{k_{j}}}\left(w^{n_{k_{j}}}\right)\right\|+\frac{1+\bar{L}}{1-\bar{L}}\left\|w^{n_{k_{j}}}-x^{n_{k_{j}}}\right\| \\
& =\frac{2}{1-\bar{L}}\left\|x^{n_{k_{j}}}-w^{n_{k_{j}}}\right\|+\left\|w^{n_{k_{j}}}-\bar{S}_{n_{k_{j}}}\left(w^{n_{k_{j}}}\right)\right\| \\
& \rightarrow 0 \text { as } j \rightarrow \infty .
\end{aligned}
$$

So $\bar{x} \in \operatorname{Fix}(S)$. Then, it follows from (e) of Proposition 1.1 that $\bar{x} \in \cap_{i=1}^{p} \operatorname{Fix}\left(S_{i}\right)$. Thus $\bar{x} \in \cap_{i=1}^{p} \operatorname{Fix}\left(S_{i}\right) \cap \operatorname{Sol}(f, C)$ letting $x^{*}=\bar{x}$ and using Step 7 , we have

$$
c=\lim _{n \rightarrow \infty}\left\|x^{n}-\bar{x}\right\|=\lim _{j \rightarrow \infty}\left\|x^{n_{k_{j}}}-\bar{x}\right\|=0 .
$$

We conclude that the whole sequence $\left\{x^{n}\right\}$ converges weakly to $\bar{x} \in \cap_{i=1}^{p} \operatorname{Fix}\left(S_{i}\right) \cap \operatorname{Sol}(f, C)$. Consequently, the sequences $\left\{y^{n}\right\}$ and $\left\{w^{n}\right\}$ also converge weakly to $\bar{x}$.

Step 9. We claim that the sequences $\left\{x^{n}\right\},\left\{y^{n}\right\}$ and $\left\{w^{n}\right\}$ converge weakly to $\bar{x}$, where $\bar{x}=\lim _{n \rightarrow \infty} \operatorname{Pr}_{\cap_{i=1}^{p} \operatorname{Fix}\left(S_{i}\right) \cap \operatorname{Sol}(f, C)}\left(x^{n}\right)$. 
By Step 8, we suppose that $t^{n}:=\operatorname{Pr}_{\cap_{i=1}^{p} \operatorname{Fix}\left(S_{i}\right) \cap \operatorname{Sol}(f, C)}\left(x^{n}\right)$ and $x^{n} \rightarrow \bar{x}$ as $n \rightarrow \propto$. Using the definition of $\operatorname{Pr}_{C}(\cdot)$, we have

$$
\left\langle t^{n}-x^{n}, t^{n}-x\right\rangle \leq 0, \quad \forall x \in \cap_{i=1}^{p} \operatorname{Fix}\left(S_{i}\right) \cap \operatorname{Sol}(f, C) .
$$

It follows from Step 7 that

$$
\left\|x^{n+1}-x^{*}\right\| \leq\left\|x^{n}-x^{*}\right\|, \quad \forall n \geq 0, \quad x^{*} \in \cap_{i=1}^{p} \operatorname{Fix}\left(S_{i}\right) \cap \operatorname{Sol}(f, C) .
$$

By Lemma 3.1, we have

$$
t^{n}=\operatorname{Pr}_{\cap_{i=1}^{p} \operatorname{Fix}\left(S_{i}\right) \cap \operatorname{Sol}(f, C)}\left(x^{n}\right) \rightarrow x_{1} \in \cap_{i=1}^{p} \operatorname{Fix}\left(S_{i}\right) \cap \operatorname{Sol}(f, C) \text { as } n \rightarrow \infty \text {. }
$$

Pass the limit in (3.26) and combining this with (3.27), we have

$$
\left\langle x_{1}-\bar{x}, x_{1}-x\right\rangle \leq 0, \quad \forall x \in \cap_{i=1}^{p} \operatorname{Fix}\left(S_{i}\right) \cap \operatorname{Sol}(f, C) .
$$

This means that $\bar{x}=x_{1}$ and

$$
\bar{x}=\lim _{n \rightarrow \infty} \operatorname{Pr}_{\cap_{i=1}^{p} \operatorname{Fix}\left(S_{i}\right) \cap \operatorname{Sol}(f, C)}\left(x^{n}\right) .
$$

It follows from Step 8 that the sequences $\left\{x^{n}\right\},\left\{y^{n}\right\}$ and $\left\{w^{n}\right\}$ converge weakly to $\bar{x}$, where

$$
\bar{x}=\lim _{n \rightarrow \infty} \operatorname{Pr}_{\cap_{i=1}^{p} \operatorname{Fix}\left(S_{i}\right) \cap \operatorname{Sol}(f, C)}\left(x^{n}\right) .
$$

The proof is completed.

\section{Application to variational inequalities}

Let $C$ be a nonempty closed convex subset of $\mathcal{H}$ and $F$ be a function from $C$ into $\mathcal{H}$. In this section, we consider the variational inequalitiy problem which is presented as follows

Find $\bar{x} \in C$ such that $\langle F(\bar{x}), x-\bar{x}\rangle \geq 0 \quad$ for all $x \in C . \quad \operatorname{VI}(F, C)$

Let $f: C \times C \rightarrow \mathcal{R}$ be defined by $f(x, y)=\langle F(x), y-x\rangle$. Then problem $P(f, C)$ can be written in $V I(F, C)$. The set of solutions of $V I(F, C)$ is denoted by $\operatorname{Sol}(F, C)$. Recall that the function $F$ is called

- monotone on $C$ if

$$
\langle F(x)-F(y), x-y\rangle \geq 0, \quad \forall x, y \in C ;
$$

- pseudomonotone on $C$ if

$$
\langle F(y), x-y\rangle \geq 0 \Rightarrow\langle F(x), x-y\rangle \geq 0, \quad \forall x, y \in C ;
$$

- Lipschitz continuous on $C$ with constants $L>0$ (shortly, $L$-Lipschitz continuous) if

$$
\|F(x)-F(y)\| \leq L\|x-y\|, \quad \forall x, y \in C .
$$

Since

$$
\begin{aligned}
y^{k} & =\arg \min \left\{f\left(x^{k}, y\right)+\frac{\beta}{2}\left\|y-x^{k}\right\|^{2}: y \in C\right\} \\
& =\arg \min \left\{\left\langle F\left(x^{k}\right), y-x^{k}\right\rangle+\frac{\beta}{2}\left\|y-x^{k}\right\|^{2}: y \in C\right\} \\
& =\operatorname{Pr}_{C}\left(x^{k}-\frac{1}{\beta} F\left(x^{k}\right)\right),
\end{aligned}
$$


Algorithm 2.1, the convergence algorithm for finding a common element of the set of common fixed points of $p$ strict pseudocontractions and the set of solutions of equilibrium problems for pseudomonotone bifunctions is presented as follows:

Algorithm 4.1 Give a tolerance $\varepsilon>0$. Choose $x^{0} \in C, k=0, \gamma \in(0,1), 0<\sigma<\frac{\beta}{2}$ and positive sequences $\left\{\lambda_{n, i}\right\}$ and $\left\{\alpha_{n}\right\}$ satisfy the conditions:

$$
\left\{\begin{array}{l}
\left\{\alpha_{n}\right\} \subset[a, b] \subset(\bar{L}, 1) \text { where } \bar{L}:=\max \left\{L_{i}: \quad 1 \leq i \leq p\right\}, \\
\sum_{i=1}^{p} \lambda_{n, i}=1 \text { forall } n \geq 1, \lim _{n \rightarrow \infty} \lambda_{n, i}=\lambda_{i} \in(0,1) \text { for all } i=1, \ldots, p, \sum_{i=1}^{p} \lambda_{i}=1 .
\end{array}\right.
$$

\section{Step 1. Compute}

$$
\gamma^{k}=\operatorname{Pr}_{C}\left(x^{k}-\frac{1}{\beta} F\left(x^{k}\right)\right) \text { and set } r\left(x^{n}\right)=x^{n}-y^{n} .
$$

If $\left\|r\left(x^{n}\right)\right\| \neq 0$ then go to Step 2. Otherwise, set $w^{n}=x^{n}$ and go to Step 3 .

Step 2. (Armijo-type linesearch techniques) Find the smallest positive integer number $m_{n}$ such that

$$
\left(1-\gamma^{m_{n}}\right)\left\langle F\left(x^{n}-\gamma^{m_{n}} r\left(x^{n}\right)\right), r\left(x^{n}\right)\right\rangle \geq \sigma\left\|r\left(x^{n}\right)\right\|^{2} .
$$

\section{Compute}

$$
w^{n}=\operatorname{Pr}_{C \cap H_{n}}\left(x^{n}\right),
$$

where $z^{n}=x^{n}-\gamma^{m_{n}} r\left(x^{n}\right)$ and $H_{n}=\left\{x \in \mathcal{H}:\left\langle F\left(z^{n}\right), x-z^{n}\right\rangle \leq 0\right\}$, and go to Step 3 .

\section{Step 3. Compute}

$$
x^{n+1}=\alpha_{n} w^{n}+\left(1-\alpha_{n}\right) \sum_{i=1}^{p} \lambda_{n, i} S_{i}\left(w^{n}\right) .
$$

Increase $n$ by 1 and go back to Step 1 .

Using Theorem 3.2, we also have the convergence of Algorithm 4.1 as the follows:

Theorem 4.2 Let $C$ be a nonempty closed convex subset of $\mathcal{H}$. Let $F: C \rightarrow \mathcal{H}$ be continuous and pseudomonotone, and $S_{i}: C \rightarrow C$ be a $L_{i}$-Lipschitz pseudocontractions for all $i=1, \ldots, p$ such that $\cap_{i=1}^{p} \operatorname{Fix}\left(S_{i}\right) \cap \operatorname{Sol}(f, C) \neq \emptyset$. Then the sequences $\left\{x^{n}\right\},\left\{y^{n}\right\}$ and $\left\{w^{n}\right\}$ generated by Algorithm 4.1 converge weakly to the point $x^{*}$, where $x^{*}=\lim _{n \rightarrow \infty} \operatorname{Pr}_{\cap_{i=1}^{p}} \operatorname{Fix}\left(S_{i}, C\right) \cap \operatorname{Sol}(F, C)\left(x^{n}\right)$.

\section{Acknowledgements}

The work was supported by the Vietnam National Foundation for Science Technology Development (NAFOSTED).

\section{Author details}

${ }^{1}$ Department of Scientific Fundamentals, Posts and Telecommunications Institute of Technology, Hanoi, Vietnam

${ }^{2}$ Department of Natural Sciences, Duy Tan University, Danang, Vietnam

\section{Authors' contributions}

The main idea of this paper is proposed by PNA. All authors read and approved the final manuscript.

\section{Competing interests}

The authors declare that they have no competing interests.

Received: 2 January 2012 Accepted: 10 May 2012 Published: 10 May 2012

\section{References}

1. Anh, PN: A logarithmic quadratic regularization method for solving pseudomonotone equilibrium problems. Acta Mathematica Vietnamica. 34, 183-200 (2009) 
2. Anh, PN: An LQP regularization method for equilibrium problems on polyhedral. Vietnam J. Math. 36, 209-228 (2008)

3. Anh, PN, Kim, JK, Nam, JM: Strong convergence of an extragradient method for equilibrium problems and fixed point problems. J. Korean Math. Soc. 49, 187-200 (2012). doi:10.4134/JKMS.2012.49.1.187

4. Blum, E, Oettli, W: From optimization and variational inequality to equilibrium problems. Math. Student. 63, 127-149 (1994)

5. Mastroeni, G: Gap function for equilibrium problems. J. Global Optim. 27, 411-426 (2004)

6. Peng, JW: Iterative algorithms for mixed equilibrium problems, strict pseudocontractions and monotone mappings. J. Optim. Theory Appl. 144, 107-119 (2010). doi:10.1007/s10957-009-9585-5

7. Acedo, GL, Xu, HK: Iterative methods for strict pseudo-contractions in Hilbert spaces. Nonlinear Anal. 67, 2258-2271 (2007). doi:10.1016/.jna.2006.08.036

8. Takahashi, S, Takahashi, W: Viscosity approximation methods for equilibrium problems and fixed point problems in Hilbert spaces. J. Math. Anal. Appl. 331, 506-515 (2007). doi:10.1016/j.jmaa.2006.08.036

9. Ceng, LC, Petrusel, A, Lee, C, Wong, MM: Two extragradient approximation methods for variational inequalities and fixed point problems of strict pseudo-contractions. Taiwanese J. Math. 13, 607-632 (2009)

10. Anh, PN, Kim, JK: Outer approximation algorithms for pseudomonotone equilibrium problems. Comput. Math. Appl. 61 , 2588-2595 (2011). doi:10.1016/j.camwa.2011.02.052

11. Anh, PN, Muu, LD, Nguyen, VH, Strodiot, Jj: Using the Banach contraction principle to implement the proximal point method for multivalued monotone variational inequalities. J. Optim. Theory Appl. 124, 285-306 (2005). doi:10.1007/ s10957-004-0926-0

12. Anh, PN, Son, DX: A new iterative scheme for pseudomonotone equilibrium problems and a finite family of pseudocontractions. J. Appl. Math. Inf. 29, 1179-1191 (2011)

13. Ceng, LC, Al-Homidan, S, Ansari, QH, Yao, JC: An iterative scheme for equilibrium problems and fixed point problems of strict pseudo-contraction mappings. J. Comput. Appl. Math. 223, 967-974 (2009). doi:10.1016/j.cam.2008.03.032

14. Kumama, P, Petrot, N, Wangkeeree, R: A hybrid iterative scheme for equilibrium problems and fixed point problems of asymptotically k-strict pseudo-contractions. J. Comput. Appl. Math. 233, 2013-2026 (2010). doi:10.1016/j.cam.2009.09.036

15. Nadezhkina, N, Takahashi, W: Weak convergence theorem by an extragradient method for nonexpansive mappings and monotone mappings. J. Optim. Theory Appl. 128, 191-201 (2006). doi:10.1007/s10957-005-7564-z

16. Peng, JW, Yao, JC: Ishikawa iterative algorithms for a generalized equilibrium problem and fixed point problems of a pseudo-contraction mapping. J. Global Optim. 46, 331-345 (2010). doi:10.1007/s10898-009-9428-9

17. Wang, S, Guo, B: New iterative scheme with nonexpansive mappings for equilibrium problems and variational inequality problems in Hilbert spaces. J. Comput. Appl. Math. 233, 2620-2630 (2010). doi:10.1016/j.cam.2009.11.008

18. Yao, Y, Liou, YC, Wu, YJ: An extragradient method for mixed equilibrium problems and fixed point problems. Fixed Point Theory Appl. Volume (2009). Article ID 632819, 15 pages, doi:10.1155/2009/632819

19. Zeng, LC, Yao, JC: Strong convergence theorem by an extragradient method for fixed point problems and variational inequality problems. Taiwanese J. Math. 10, 1293-1303 (2010)

20. Daniele, P, Giannessi, F, Maugeri, A: Equilibrium problems and variational models. Kluwer Academic Publishers, Dordrecht (2003)

21. Takahashi, S, Toyoda, M: Weakly convergence theorems for nonexpansive mappings and monotone mappings. J. Optim. Theory Appl. 118, 417-428 (2003). doi:10.1023/A:1025407607560

doi:10.1186/1687-1812-2012-82

Cite this article as: Anh and Hien: The extragradient-Armijo method for pseudomonotone equilibrium problems and strict pseudocontractions. Fixed Point Theory and Applications 2012 2012:82.

\section{Submit your manuscript to a SpringerOpen ${ }^{\circ}$ journal and benefit from:}

- Convenient online submission

- Rigorous peer review

- Immediate publication on acceptance

- Open access: articles freely available online

- High visibility within the field

- Retaining the copyright to your article

Submit your next manuscript at $\boldsymbol{\nabla}$ springeropen.com 